\title{
Optimization of Complex Multi-Band Joint Spectral Measurements of Ambiant Media Objects
}

\author{
H.H. Asadov, Sh. N. Jahidzadeh
}

\begin{abstract}
The paper is devoted to questions on optimization of comlex multi-band joint spectral measurements of environments objects. It is shown that results of joint multi-spectral measurements could be assessed by new indicator of authenticity expressed by signal-noise ratio at the output of spectral channels of multispectral measuring instrument.

Summing of such indicator on all spectral channels and substitute of researched object by model of ideal black body characterized by Plunk function make it possible to derive the formulae of optimal interrelation between mean quadratic deviation of noises and model function. On the basis of said optimum interrelation function the new optimum interrelation function concerning dependence of mean quadratic deviation of noises on wavelength and absolute temperature is derived. It is shown that in optimum regime, upon some value of wavelength the mean quadratic deviation can reach the maximum amount i.e. the authenticity of measuring results in this case would be minimum.
\end{abstract}

Index Terms - joint measurements, multispectral measurements, optimization, absolute black body, absorption coefficient.

\section{INTRODUCTION}

It is well known that the joint measurements are such ones where values of measured parameters are should be determined in line with results of measurements of similar parameters upon their various links and relations. [1].Values of measured parameters are determined by solution set of equations composed in line with physical laws and regularities chosen as a basis of carried out measurements.

As regard joint multi-spectral atmospheric measurements the following simple example may be considered. Assume that one should measure total (column) amount of gases $M_{1}$ и $M_{2}$ with absorption coefficients $\alpha_{1}(\lambda)$ и $\alpha_{2}(\lambda)$. At the wavelengths $\lambda_{1}$ и $\lambda_{2} \quad$ we have

$$
\begin{aligned}
& \alpha_{1}\left(\lambda_{1}\right) \neq \alpha_{2}\left(\lambda_{1}\right) \\
& \alpha_{1}\left(\lambda_{2}\right) \neq \alpha_{2}\left(\lambda_{2}\right)
\end{aligned}
$$

At the same time:

$\alpha_{1}\left(\lambda_{1}\right) \neq \alpha_{1}\left(\lambda_{2}\right)$

$\alpha_{2}\left(\lambda_{1}\right) \neq \alpha_{2}\left(\lambda_{2}\right)$

In this case upon carrying out measurements at the wavelength $\lambda_{1}$ in line with Bouguer-Beer law following

H.H. Asadov, doctor of technical sciences, professor, head of department of Research institute of Aerospace Informatics of National Aerospace Agency of Azerbaijan Republic

Professor Sh. N. Jahidzadeh, doctorate of National Aerospace Agency, Baku, Azerbaijan Republic equation could be written

$$
\begin{aligned}
& I_{1}\left(\lambda_{1}\right)=I_{0}\left(\lambda_{1}\right) \exp \left[-\left[\alpha_{1}\left(\lambda_{1}\right) \cdot m_{1}+\right.\right. \\
& \left.\left.\alpha_{2}\left(\lambda_{1}\right) \cdot m_{2}\right]\right]
\end{aligned}
$$

At the same time the measurements at the wavelength $\lambda_{2}$ are carried out. Similar to equation (3) following formulae could be written

$$
\begin{aligned}
& I_{2}\left(\lambda_{2}\right)=I_{0}\left(\lambda_{2}\right) \exp \left[-\left[\alpha_{1}\left(\lambda_{2}\right) \cdot m_{1}+\right.\right. \\
& \alpha 2 \lambda 2 \cdot m 2,
\end{aligned}
$$

Where in equations (3) and (4) $I_{1}\left(\lambda_{1}\right)$ and $I_{2}\left(\lambda_{2}\right)-$ signals at the outputs of the first and second measuring instruments carrying out measurements at the wavelengths $\lambda_{1}$ и $\lambda_{2} ; I_{0}\left(\lambda_{1}\right)$ и $I_{0}\left(\lambda_{2}\right)$ - luminous fluxes of used sources of illumination emitting at the wavelengths $\lambda_{1}$ и $\lambda_{2}$

Joint solution of equations (3) и (4) regarding $m_{1}$ и $m_{2}$ make it possible to calculate these parameters.

Some real examples for utilization of joint multi-spectral measurements could be considered. The best example is International set of aerosol measurements «AERONET», containing more than 500 automated stations for measurements of atmospheric aerosol optical depth at the wavelengths 340, 440, 500, 670, 870, $1020 \mathrm{~nm}$ [2].

Other example for joint measurements is remote measurements of soil pollution carried out in ultraviolet, visible and infrared bands [3, 4], and also the method for detection of hydrocarbons fire in ultraviolet and infrared bands[5].

Thus it may be concluded that joint multispectral measurements are inseparable part of theory and practice of multispectral measurements and optimization of them is an actual task.

\section{SUGGESTED METHOD}

One of major parameters of any measuring process is signal-noise ratio at the input of measuring instrument $\psi$, determined as

$\psi\left(\lambda_{2}\right)=\frac{f(\lambda)}{\sigma(\lambda)}$

where: $f(\lambda)$-amplitude of measuring signal at the input of measuring channel carrying out measurements at the wavelength $\lambda$;

$\sigma(\lambda)$-m.s.d. of noises at the input of measuring channel of 
instrument carrying out measurements at the wavelength $\lambda$. Following parameter may be suggested as probability type measure of authenticity of result of carried out measurements

$$
\begin{gathered}
P(\lambda)=1-\frac{1}{\psi(\lambda)} ; \\
\psi(\lambda) \geq 1
\end{gathered}
$$

Carried out joint multispectral measurements at the wavelengths $\lambda_{i}, \quad i=\overline{1, n}$; could be characterized by following mean parameter

$$
P_{c p . g}=\frac{1}{n} \sum_{i=1}^{n}\left[1-\frac{1}{\psi\left(\lambda_{i}\right)}\right]
$$

Taking into account formulas (5) and (7) we get

$$
P_{c p . g}=\frac{1}{n} \sum_{i=1}^{n}\left[1-\frac{\sigma\left(\lambda_{i}\right)}{f\left(\lambda_{i}\right)}\right]
$$

Assume that following limitation condition does exist

$$
\sum_{i=1}^{n} f\left(\lambda_{i}\right)=\mathrm{C}_{1} ; \quad \mathrm{C}_{1}=\text { const }
$$

(9)

In this case the discrete variation task may be composed for determination of optimum interrelation between $f\left(\lambda_{i}\right)$ and $\sigma\left(\lambda_{i}\right)$ upon which the target functional could reach the extremum. Taking into account formulas (8) and (9) we get $P_{g . o}=$

$\frac{1}{n} \sum_{i=1}^{n}\left[1-\frac{\sigma\left(\lambda_{i}\right)}{f\left(\lambda_{i}\right)}\right]-\gamma\left[\sum_{i=1}^{n} f\left(\lambda_{i}\right)-C\right]$

where $\gamma$-Lagrange multiplier.

In continual form the functional (10) may be written as follows

$P_{\mathrm{H} . \mathrm{o}}=\int_{\lambda_{\min }}^{\lambda_{\max }}\left[1-\frac{\sigma(\lambda)}{f(\lambda)}\right] d \lambda-$

$\gamma\left[\int_{\lambda_{\min }}^{\lambda_{\max }} f(\lambda) d \lambda-C_{2}\right]$

Where

$\int_{\lambda_{\min }}^{\lambda_{\max }} f(\lambda) d \lambda=C_{2} ; \quad C_{2}=$ const

(12)

To proceed with solution of optimization task (11), (12) it should be noted that in multispectral measurements of ambient media objects researchers often use sources of illumination which could be well approximated by Plank function written as follows [6].

$\rho_{\lambda, T}=\frac{8 \pi h c}{\lambda^{5}} \cdot \frac{1}{e^{h c / \lambda k T}-1}$

where $\rho$ - voluminous density of irradiation i.e. energy of irradiation in single measure of volume;

$$
\begin{gathered}
h \text { - Plank constant; } \\
k \text { - Boltzmans constant; } \\
c \text { - speed of light; }
\end{gathered}
$$

$\lambda$ - wavelentgh;

$T$-absolute temperature, $\mathrm{K}$.

Meanwhile according to Stephane-Boltzmann law full emission capability of black body is determined as

$v_{\lambda}=\int_{0}^{\infty} \rho_{\lambda, T} d \lambda=\sigma \cdot T^{4}$

where: $\sigma$-Stephane-Boltzmann constant.

Thus regarding multispectral measuring systems when $\lambda_{\min }=0 ; \lambda_{\max }=\infty$ the condition (12) is same with (14) upon $T=$ const.

Henceforth it could be concluded that if researched object has a constant temperature all procedure of multispectral measurements of such objects could be optimized by solution of optimization task (11), (12).

Solution of optimization $\operatorname{task}(11)$, (12) is as follows. According to Euyler- Lagrange equation the solution of the task should meet following condition

$$
\frac{d\left\{\left[1-\frac{\sigma(\lambda)}{f(\lambda)}\right]+\gamma\left[f(\lambda)-C_{2}\right]\right\}}{d f(\lambda)}=0
$$

(15)

From formula (15) we get

$$
\frac{\sigma(\lambda)}{f^{2}(\lambda)}-\gamma=0
$$

From formula we have

$f(\lambda)=\sqrt{\frac{\sigma(\lambda)}{\gamma}}$

Taking into account formulas (12) and (17) we get

$\int_{0}^{\infty} \sqrt{\frac{\sigma(\lambda)}{\gamma}} d \lambda=C_{2}$

From formula (18) we have

$$
\left[\frac{1}{C_{2}} \cdot \int_{0}^{\infty} \sqrt{\sigma(\lambda)} d \lambda\right]^{2}=\gamma
$$

From formulas (17) and(19) we have

$$
f(\lambda)=\frac{\sqrt{\sigma(\lambda)} \cdot C_{2}}{\int_{0}^{\infty} \sqrt{\sigma(\lambda)} d \lambda}
$$

Therefore upon interrelation between $f(\lambda)$ and $\sigma(\lambda)$ characterized by function (20) the target functional reaches the extremum value. In order to determine the minimum type of extremum the second derivative of integrant in (11) on $f(\lambda)$ should be calculated and its negative value could be revealed. Thus the functional (11) upon solution (20) reaches its maximum.

The next point of researches is following question- upon what type of function $\sigma(\lambda)$ multispectral measurements could be identified as of optimum procedure if the researched object is absolute black body? Assume

$$
f(\lambda)=\rho_{\lambda, T} .
$$

Taking into accountc (13) и (20) we get 
$\frac{8 \pi h c}{\lambda^{5}} \cdot \frac{1}{e^{h c / \lambda k T-1}}=\frac{\sqrt{\sigma(\lambda)} \cdot C_{2}}{\int_{0}^{\infty} \sqrt{\sigma(\lambda)} d \lambda}$

The formula (21) is equation solution of which make it possible to determine the optimum function $\sigma(\lambda)$, providing for maximum authenticity of multispectral measurements of objects well approximated by absolute black body characteristics.

Solution of equation (21) as follows. Assume

$\int_{0}^{\infty} \sqrt{\sigma(\lambda)} d \lambda=C_{3}$

From formulas (21) and (22) we get

$$
\sigma(\lambda)=\left[\frac{8 \pi h c \cdot C_{3}}{\lambda^{5} \cdot C_{2}} \cdot \frac{1}{e^{h c / \lambda k T}}\right]^{2}
$$

or

$$
\sigma(\lambda)=C_{4} \cdot \lambda^{-10} \cdot e^{-h c / \lambda k T}
$$

Thus as it follows from formula (24) the optimum function $\sigma(\lambda)$ may has an extremum on $\lambda$.

Researching extremum of formula (24) on $\lambda$ we get

$$
\begin{aligned}
& \frac{d \sigma(\lambda)}{d \lambda}=-10 \cdot \lambda^{-11} \cdot C_{4} \cdot \\
& e^{-2 h c / \lambda k T}+C_{4} \cdot \lambda^{-10} \cdot e^{-2 h c / \lambda k T} .
\end{aligned}
$$

$\frac{2 h c}{\lambda^{2} k T}$

Upon condition

$$
\frac{d \sigma(\lambda)}{d \lambda}=0
$$

It could be found from(25) that

$$
\lambda^{-1}=10^{-1} \cdot \frac{2 h c}{\lambda^{2} k T}
$$

or

$$
\lambda=\frac{h c}{5 k T}
$$

It can be shown that upon solution (27) the function (24) reaches its maximum value i.e. the wavelength chosen in line with formula (27) will provide the minimum authenticity of results of multispectral joint measurements of objects which can be well approximated by ideal black body characteristics.

\section{CONCLUSION}

Thus it is shown that results of complex joint multispectral measurements could be characterized by suggested new probability type indicator of authenticity, determined by signal-noise ratio at the output of spectral measuring channel. Integration of suggested indicator on all measuring spectral channels and approximation of researched objects by characteristics of ideal black body i.e. Plank function make it possible to obtain the formula of optimum interrelation between m.s.d. of noises and Plank function. On the basis of calculated optimum interrelation function the analytical dependence of m.s.d. of noises on wavelength and absolute temperature is obtained. It is shown that upon some value of wavelength m.s.d. of noises could reach the maximum value i.e. authenticity of results of measurements could reach its minimum.

\section{REFERENCES}

[1] V.I. Kolchkov. Metrologija standartizacija i sertifikacija. «Mashinostroenie», M. 2015, 410s (in Russian)

[2] B. N. Holben, T. F. Eck, I. Slutsker, D. Tanre, J. P. Buis, A. Setzer, E. Vermote, J. A. Reagan, Y. J. Kaufman, T. Nakajima, F. Lavenu, I. Jankowiak, and A. Smirnov. AERONET-A Federated Instrument Network and Data Archive for Aerosol Characterization.

[3] International Journal of GEOMATE, Nov., 2017, Vol.13, Issue 39, pp. 33-35. Moses E. Emetere, Samuel E. Sanni, Jennifer M. Emetere, and Uno E. Uno THERMAL INFRARED REMOTE SENSING OF HYDROCARBON IN LAGOS- SOUTHERN NIGERIA: APPLICATION OF THE THERMOGRAPHIC MODEL .

[4] G.Andreoli, B. Bulgarelli, B. Hosgood, D.Tarchi Hyperspectral Analysis of Oil and Oil - Impacted Soils for Remote Sensing Purposes.

[5] J.W. Hoffman, J.A. Brass Estimating Fire Properties by Remote Sensing.

[6] Zh. Gossorg. Infrakrasnaja termografija. M. «Mir». 1988. 399 s (in Russian) 